\title{
'Malnutrition' in the aged: the dietary assessment
}

\author{
Mark L Wahlqvist* \\ Asia Pacific Health \& Nutrition Centre, Monash Asia Institute, PO Box 1 1A, Monash University, Melbourne, \\ Victoria 3800, Australia
}

\begin{abstract}
Changing demography towards older populations requires nutritionists to review food-health relationships in regard to older age groups, to the ageing process, to precursors of disability and survival, and to the changeability of health status through diet in later life. Nutritional status in later life may be complex with combined features of under-, over- and disordered nutrition - dysnutrition. Further, beyond food components (essential nutrients and more), foods themselves, integrals of food intake and the socio-cultural aspects of food intake play a significant role in the health and well-being of the aged and require measurement. Ageing socio-biology provides opportunities and imposes limitations on the usual food intake instruments. However, rapid assessment procedures can be particularly valuable as highlighted in the Food Habits in Later Life project of the International Union of Nutritional Sciences. There are critical issues to which dietary methodology can be applied to the aged: a disappearing knowledge base, relevant health-enhancing strategies and the creation of new food-health opportunities. Many communities now wish to use such information to develop food-based dietary guidelines for their aged members.
\end{abstract}

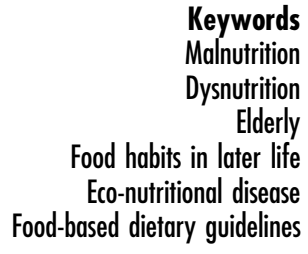

Nutritional disorder in the aged may be very complex, not simply 'under-' or 'over-', or deficient in energy, macro- or micronutrients. 'Dysnutrition' may be a better term as it comes with fewer assumptions ${ }^{1}$. Food intake is one, but not the only, determinant of dysnutrition in the aged ageing, disease and its treatment also contribute. Thus, the focus of food in preventing or managing dysnutrition may be on intake, body composition (e.g. sarcopenia, fatness or osteopenia), a function or a disease process.

\section{The value of dietary information about the aged}

While description of food practices and intakes among the aged are worthwhile in their own right for historical purposes, the changing demography of the human population, towards one that is more aged, requires a critical analysis of food-health relationships in this age group so as to enable healthy ageing to occur. The interest among policy makers, in the community itself and from an economic point of view in food and ageing is now quite intense. It goes well beyond the narrow confines of wasting among hospitalised and institutionalised aged people. The nutritional status of this group may be complex, even by the traditional analysis of energy, macro- and micronutrients, let alone other food components of biological relevance. As well, the social role of food in health must not be neglected in the assessment.

\section{Predicting health outcomes}

A knowledge of what different communities of older people and different old individuals eat, with tracking over various periods of time, allows models to be developed to identify preferred food patterns, foods and food components. While, in the minds of many biomedical scientists, this falls short of the more definitive nature of clinical nutrition trials, the modelling approach will be utilised more and more, because it is integrative, more timely, less costly and more practical.

Clinical trials addressing at best two, or three variables at once may not reach useful conclusions for many years and short-change the possibilities emerging for whole-oflife food-pattern approaches to healthy ageing. The Lyon Diet Heart Study exemplified the employment of this technique. It investigated the effect of a Mediterraneantype diet compared with a prudent Western diet on the rate of recurrence of fatal and non-fatal coronary myocardial infarction ${ }^{2,3}$. This study has proved that simple dietary changes can have a great impact on mortality.

Investigators are now modelling traditional food cultures and changes in food cultures, and examining the predictive power of these models in different geographic settings. An example for the use of modelling science in the nutrition field is a mortality follow-up study by the International Union of Nutritional Sciences (IUNS). This study has shown that the traditional Greek food pattern score can be used to predict survival. It is apparent that the relationship between diet score and survival can also be applied with Greek migrants and Anglo-Celtic Australians ${ }^{4,5}$. Modelling science in the nutrition field will become as important as is it is in the physico-chemical and engineering sciences. The exception to this view would be 
Table 1 Consequences of food choice in the aged

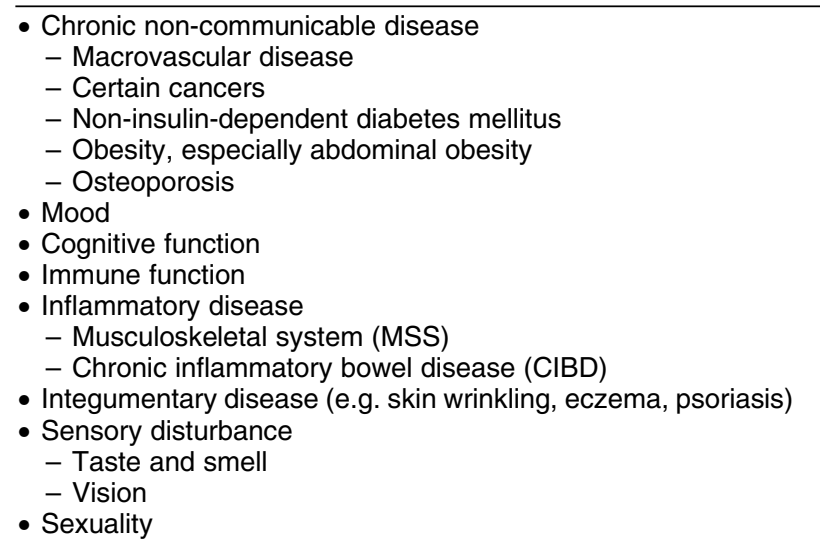

those situations in which it is possible to study, in randomised fashion, different food patterns over finite periods of time, and achieve measurable gains in health outcomes.

The full consequences of food choice have yet to be appreciated. So far, these are located around the so-called chronic non-communicable diseases (CNCDs), which are probably better referred to as 'eco-nutritional diseases' (ENDs) because they represent a problem created by changing environments and especially by the ways in which these influence our food habits. In any case, the way in which they are described affects the way in which we think about them from the preventive and management point of view, and is a challenge for those addressing the nutritional and health needs for older people. The diseases in question here are macrovascular disease, certain cancers, non-insulin-dependent diabetes, obesity, especially abdominal obesity, and osteoporosis. Other emergent health problems related to food choice are shown in Table 1.

\section{Available food intake assessment methods}

Methods available to assess food intake in later life range from those that come from the socio-anthropological sciences and include oral history (with the ability to provide narrative), focus groups and various Rapid Assessment Procedures (RAP) through to the more systematic and familiar methods of repeated 24-hour recalls, food-frequency questionnaires and dietary histories with cross-checking. The methods need to be sensitive enough to detect early and correctable warnings of health risk. The food measures in question will probably be indices. For example, RAP encourages the expression of food culture of the study communities and, within the framework of food habits inquiries, recognises the process of modification. The procedures have been used to set the framework for the various community studies of the Food Habits in Later Life (FHILL) project ${ }^{6}$.
Table 2 Food-based dietary guidelines for the aged ${ }^{9}$

1. Achieve food variety within and across food groups

2. Prefer unrefined fat from whole foods

3. Recreate traditional foods/dishes from other cuisines to increase food variety

4. Develop healthy novel foods and functional foods

5. In more urban settings, food industry to produce 'home meal replacements' or 'fast foods' that are low in animal fats

6. Understand that time, frequency and size of meals may be important

7. Take into account physical activity and level of energy throughput, with required nutrient density ${ }^{10}$

\section{Limitations for application among the aged}

The peculiar limitations of available dietary methods for application among the aged are:

- literacy;

- culture poorly understood by the observers;

- memory loss; and

- accessibility of subjects who may be isolated and have limited mobility.

Thus the investigators in this field may need to be more multi-skilled, innovative and patient than for other age groups.

\section{Applications of dietary methodology to critical issues for the aged}

The applications of dietary methodology in later life include a number of critical issues in food, nutrition and health science as it applies to the aged:

1. documentation of a disappearing knowledge base, with the provision of information for inter-generational transfer;

2. recognition and understanding of food intake patterns in later life conducive to well-being, reduced morbidity and decreased mortality; and

3. how health may be enhanced by the transfer of ideas and knowledge, food cultural synthesis, and the development of new foods and food patterns.

\section{Food-based dietary guidelines for the aged}

The World Health Organization has emphasised that dietary guidelines be food-based ${ }^{7-9}$. Food-Based Dietary Guidelines (FBDGs) have now been developed for the aged (Table 2). The guidelines go beyond addressing 'foods' simply as 'food groups' and avoid reference to nutrients at the point of guidance. At the same time, they take account of the best nutrition (including nutrients) science available. The guidelines also address the ways in which foods are produced (agriculture, horticulture), prepared (like cuisine), processed (like 
food industry) and developed (like novel, functional foods).

\section{Future directions}

The future should allow us to be clearer about ways of eating for healthy ageing; the integration of food habits with other lifestyle measures for health enhancement; and a whole-of-life approach from conception, through growth and development, the reproductive span and the later years. Through these strategies, dysnutrition among the aged can be minimised.

\section{References}

1 Wahlqvist ML, Savige GS, Lukito W. Nutritional disorders in the elderly. Med. J. Aust. 1995; 163: 376-81.

2 de Lorgeril M, Renaud S, Mamelle N, Salen P, Martin JL, Monjaud I, et al. Mediterranean alpha-linolenic acid-rich diet in secondary prevention of coronary heart disease. Lancet 1994; 343: 1454-9.

3 de Lorgeril M, Salen P, Martin JL, Monjaud I, Delaye J, Mamelle N. Mediterranean diet, traditional risk factors, and the rate of cardiovascular complications after myocardial infarction: final report of the Lyon Diet Heart Study. Circulation 1999; 99(6): 779-85.
4 Trichopoulou A, Kouris-Blazos A, Wahlqvist ML, Gnardellis C, Lagiou P, Polychronopoulos E, et al. Diet and overall survival in elderly people. Br. Med. J 1995; 311: 1457-60.

5 Kouris-Blazos A, Gnardellis C, Wahlqvist ML, Trichopoulos D, Lukito W, Trichopoulou A. Are the advantage of the Mediterranean diet transferable to other populations? A cohort study in Melbourne, Australia. Br. J. Nutr. 1999; 82: $57-61$.

6 Scrimshaw S, Hurtado E. RAP: Rapid Assessment Procedures for Nutrition and Primary Health. Los Angeles: UCLA Latin American Centre Publications, University of California, 1987.

7 World Health Organization (WHO). Preparation and Use of Food-Based Dietary Guidelines. Report of a Joint FAO/WHO Consultation on the Preparation and Use of Food-Based Dietary Guideline, Nicosia, Cyprus. Geneva: WHO, 1996.

8 World Health Organization (WHO). Preparation and Use of Food-Based Dietary Guidelines. Report of a Joint FAO/WHO Consultation on the Preparation and Use of Food-Based Dietary Guideline. WHO Technical Report Series 880 . Geneva: WHO, 1998.

9 Wahlqvist ML, Kouris-Blazos A, Savige G. Food security and the aged. In: Ogunrinade A, Oniang'o RK, May J, eds. Not by Bread Alone: Food Security and Governance in Africa. South Africa: Toda Institute for Global Peace and Policy Research, 1999; 206-21.

10 Wahlqvist ML, Flint DM. Nutrition requirements and recommended dietary intakes for the elderly - vitamins. In: Horwitz A, ed. Nutrition in the Elderly. Oxford: Oxford University Press [published on behalf of the World Health Organization], 1989; 123-36. 